\title{
Response latency in visual search with redundancy in the visual display
}

\author{
JOHN E. HOLMGREN \\ University of Maryland, College Park, Maryland 20742 \\ JAMES F. JUOLA \\ University of Kansas, Lawrence, Kansas 66044 \\ and \\ RICHARD C. ATKINSON \\ Stanford University, Stanford, California 94305
}

\begin{abstract}
Two experiments were run to investigate the effects of redundant display items upon response latency in a visual search task. In the first study, Ss searched five-letter displays for a predesignated critical letter. Both critical and noncritical letters could be repeated in the displays. Mean response latency decreased markedly with increasing redundancy in the critical letter and was affected to a lesser extent by redundancy in the noncritical letters. In the second study, Ss were required to detect the presence of redundant letters in displays of from two to five letters, first with no information as to what letter might be repeated, then with knowledge of which letter would be repeated if the display contained a redundant letter. Response latencies in the former case were much slower than in the latter. The implications of these findings for current views of visual information processing were discussed.
\end{abstract}

Estes and various co-workers (Estes \& Taylor, 1966; Wolford, Wessel, \& Estes, 1968; Bjork \& Estes, 1971; Estes, 1972) have investigated the effects of redundancy in a forced-choice visual search task. In this type of task, Ss are usually required to determine which of two critical letters is present in a tachistoscopically presented display of letters. The critical letter in a display on a given trial is considered redundant if it appears more than once. As Estes's results have clearly shown, the probability of a correct forced-choice response increases with increasing redundancy. However, these studies have also shown that when response latencies are corrected for guessing, they are constant across redundancy levels.

On the basis of an exhaustive study examining the variables previously manipulated in forced-choice studies, Estes (1972) interprets the redundancy results as supporting the view that increasing redundancy raises the probability that an instance of the critical letter will fall near the fovea within an area of maximal discriminability. This results in a fast correct response, or as Estes (1972) refers to it, a "primary detection response." If no critical item falls in this area of maximal discriminability, a relatively slow "secondary response" is made, based upon whatever partial information is available from the periphery. Estes argues that the various procedures used to correct for guessing effectively tap only these primary detection responses, therefore the corrected latencies are found to be independent of redundancy.

There are two problems involved in interpreting latency data from the task used by Estes. First, a two-alternative forced-choice task can always be treated as if it were a yes-no task. That is, a $\mathbf{S}$ can completely ignore one of the critical letters and attempt to determine whether the other critical letter appears in the display. If that letter is detected, then the $\mathrm{S}$ assumes the other critical letter was present and responds accordingly. A S's response latencies (and the effects of redundancy upon latency) may depend crucially on whether or not such a strategy is being employed. A second (and related) problem is that in a task in which a significant number of errors are made, response latencies cannot be meaningfully interpreted without some model of the search process that accounts for the production of those errors. If the model is incorrect, then the latencies may be misinterpreted.

One way to circumvent these problems is to investigate latencies in a yes-no task with a very low error rate. Atkinson, Holmgren, and Juola (1969) have used this type of task to look at the effect of number of letters, in a visual display on response latency. The Ss were shown a critical letter at the beginning of each trial. A linear array of from one to five different letters was then presented, with Ss being required to strike one response key if the critical letter was in the display (a positive response) and a second key if it was not (a negative response). Mean response latency was found to increase roughly linearly with display size for both positive and negative responses, with the two functions having about the same slope. The error rates at all display sizes were negligible. These results suggest that the visual processing involved in the Atkinson et al (1969) task includes some type of exhaustive comparison process; i.e., Ss compare all items in the display with some internal representationof the critical item regardless of whether or not the display contains a 
critical letter. In contrast, Estes's assumption of a primary detection response implies that on at least a portion of the trials a response occurs before all items are processed. The linearity found by Atkinson et al (1969) would seem to suggest a serial comparison process, but as those investigators pointed out, it is possible for a model assuming a parallel process to predict linearity also. Thus the Atkinson et al (1969) results do not necessarily conflict with Estes's (1972) conclusion that processing is basically parallel.

The purpose of the present studies is to investigate the effects of redundancy on visual information processing in a yes-no task designed to virtually eliminate error effects. It is hoped that this advantage over the forced-choice procedure should lead to a clearer interpretation of the role of redundant information in visual processing.

\section{EXPERIMENT I}

In this first study, Ss searched displays of constant size to determine the presence or absence of a critical letter. Displays varied with respect to redundancy of both the critical letter and the "noise" letters (i.e., the noncritical letters in the display). By varying the redundancy of both types of letters, it should be possible to gain a better understanding of the source of any effects. Taking the simplest view, if displays are processed in an exhaustive manner, then for a given display size response latency should be independent of redundancy. Of course; there are many ways of revising a simple exhaustive processing model to incorporate redundancy effects, but such revisions will not be considered until shown to be necessary. The model outlined by Estes (1972) predicts that latencies will decrease with increasing redundancy in the critical letter for the reasons given above. One must merely assume that enough information is obtained from each display to ensure that secondary responses are almost always correct.

\section{Method}

Subjects. The Ss were eight female Stanford University undergraduates with normal vision. Each was paid a total of $\$ 17.50$ for the 10 sessions of the experiment.

Stimuli. The display slides were prepared from photographs of capital consonants (no vowels were used) typed in a horizontal row with an IBM Executive "Registry" electric typewriter. A dollar sign was used as a delimeter on each side of the display, with no additional spaces between the signs and the display letters. Each display contained five letters; these could be all different (no redundancy), or one of the letters could be repeated once or twice (single and double redundancy, respectively). Within these three display classifications, each consonant was used approximately equally often at each serial position. All 10 possible permutations of repeated and nonrepeated letters were used within the two redundancy conditions, an equal number of slides being made for each permutation. A total of 20 different no-redundancy, 40 different single-redundancy, and 30 different double-redundancy displays were used.

Apparatus. The apparatus included an Iconix automated two-field tachistoscope. The displays were presented through a circular aperture onto a rear-projection ground-glass screen, illuminating an area $5.2 \mathrm{~cm}$ in diam. The displays measured $0.5 \mathrm{~cm}$ in height and $1.1 \mathrm{~cm}$ in length. From a line-of-sight viewing distance of about $60 \mathrm{~cm}$, the visual angle subtended by the displays was approx imately $2.2 \mathrm{deg}$.

Between stimulus exposures, the screen was illuminated by a second light source. A single pre- and postexposure field was used containing four small black dots forming a rectangle, $2.2 \mathrm{x}$ $1.3 \mathrm{~cm}$. The display always appeared centered within this rectangle. The brightness of the pre- and postexposure field, as measured by a Macbeth illuminometer, was $6.2 \mathrm{~mL}$, while display brightness averaged $7.0 \mathrm{~mL}$. Above the screen, three small colored lights were arranged in a vertical row. Below the screen, a single IEE Binaview character-display unit was used to present the critical letter on each trial.

On a table in front of $S$, three telegraph keys were arranged along an arc extending from $90^{\prime}$ clock to $120^{\prime}$ clock. The keys were positioned so that an $S$ could rest her right arm comfortably on the table, with her right forefinger on the center key. By moving her hand along an arc of 1 in. in either direction, the $S$ could depress either of the two response keys.

Procedure. The Ss were assigned randomly to two groups. Those in Group 1 registered a positive response (indicating a match between the critical letter and one of the display letters) by depressing the key nearest to her, and a negative response by depressing the key nearest the display. These conditions were reversed for the Ss in Group 2.

The displays were presented in a different random order for each $S$ and for each session, with the constraint that double-, single-, and no-redundancy slides would be shown in a $2: 3: 1$ ratio, respectively. The critical letter was chosen randomly from the set of undisplayed elements on negative trials and randomly from the set of displayed elements on positive trials (with the constraint that the five serial positions would be tested equally of ten within each of the three redundancy classifications). The effect of this latter constraint on displays (other than those of the no-redundancy type) was that the repeated letters were used as critical item s either two or three times as of ten as any one of the nonrepeated display letters. Therefore, on $3 / 5$ of the positive trials, the critical letter appeared once in the display, on 1/5 of the trials, it appeared twice, and on $1 / 5$ of the trials, it appeared three times. Moreover, among the positive trials with the critical letter appearing only once, $5 / 18$ had no repeated noise letters (no redundancy), 1/2 had one noise letter repeated once (single redundancy), and $2 / 9$ had one noise letter repeated twice (double redundancy). For negative trials, $1 / 6$ were no-redundancy trials, $1 / 2$ were single-redundancy trials, and $1 / 3$ were double-redundancy trials. The conditional probability that the critical letter would be present in the display, given that the display was a member of any one of the three redundancy classifications, was always equal to $1 / 2$. The sequence of positive and negative trials was random, with the restriction that there was an equal number of positive and negative trials during each session.

The following sequence of events occurred on each trial: (a) A single letter was presented for $2 \mathrm{sec}$ on the Binaview unit. (b) When the unit was turned off, $S$ pushed a button held in her left hand and, after a .5-sec delay, the display was presented for $400 \mathrm{msec}$. (c) Using her right hand, $S$ then made the appropriate response within a 2 -sec time limit after the onset of the display. (d) At the end of this period, one of the three lights above the screen was turned on for $2 \mathrm{sec}$; a green light indicated a correct response, a red light indicated an error, and a white light indicated no response or a response made after the 2-sec limit. After a .5-sec intertrial interval, a new critical letter was presented on the Binaview unit to start the next trial. Each trial lasted between 7 and $8 \mathrm{sec}$, depending on the elapsed time between the offset of the Binaview unit and the pressing of the start button by $S$.

The onset of the display triggered two latency counters, one 
stopping when the $S$ lifted her finger from the center key and the other stopping when either the positive or negative response key was struck. In this way, measures of release latencies (time from onset of display to release of center key) and terminal latencies (time from onset of display to striking of a response key) were obtained.

The Ss were run for a total of 226 trials in each session. The first 10 trials of each session were practice trials and were not recorded for subsequent analy sis. With a 5 -min rest period after the first 118 trials, a session lasted about $40 \mathrm{~min}$. All Ss participated in 10 sessions. Before each session, Ss were instructed not to release the center key until they were certain of the correct response, and then to depress the appropriate key as rapidly as possible while being careful not to make èrors. After each session, $S$ received feedback from $E$ about her performance to insure rapid responding and a low error rate.

\section{Results}

In order to minimize practice effects, the data from the first two sessions were discarded. All analyses were performed on the data from Sessions 3 through 10. While overall mean latency declined steadily over these sessions, there were no systematic changes in the redundancy effects.

The data of primary interest are mean latencies of correct responses as a function of redundancy level and response type (i.e., positive or negative). Positive trials may be separated into those having a redundant critical letter and those having a redundant noise letter. These means were obtained for each $S$, and since the data for all Ss showed the same general trends, the means for individuals were averaged across the eight Ss. These latter values are shown in Fig. 1. While for most Ss, there were no important differences between trends for release latencies and those for terminal latencies, only terminal latencies are presented here since at least one $S$ appeared to be releasing the center key prematurely on a significant proportion of the trials.

Mean latencies for positive trials decrease significantly with increasing redundancy in the critical letter $(F=$

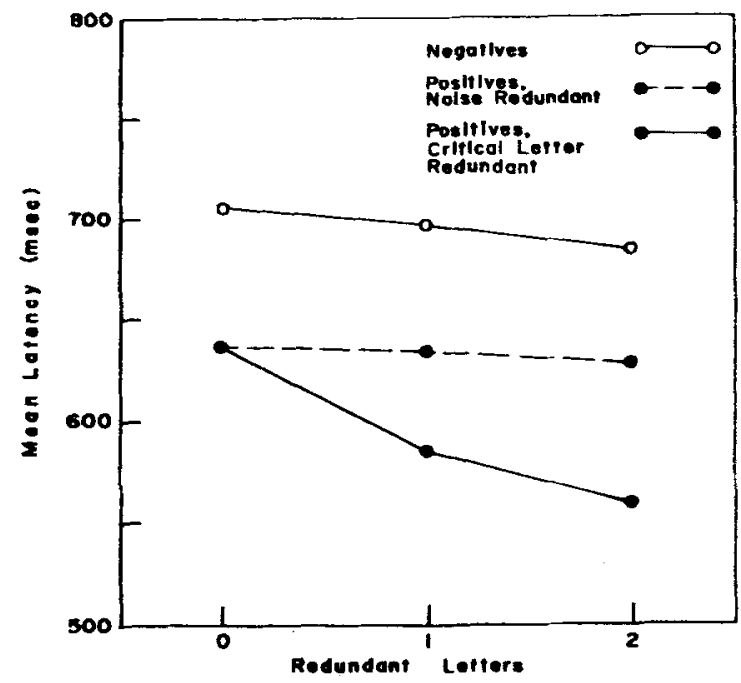

Fig. 1. Mean latency of positive and negative responses for Experiment I (averaged over Ss and Sessions 3-10) as a function of redundancy level.
Table 1

Mean Latency (in Milliseconds) as a Function of Serial Position of Critical Letters

\begin{tabular}{|c|c|c|c|c|c|}
\hline \multicolumn{6}{|c|}{ Redundancy } \\
\hline \multicolumn{2}{|c|}{ None } & \multicolumn{2}{|c|}{ Single } & \multicolumn{2}{|c|}{ Double } \\
\hline $8 P^{*}$ & ML & SP* & ML & SP* & ML \\
\hline 1 & 592 & 12 & 578 & 123 & 548 \\
\hline 2 & 656 & 13 & 562 & 124 & 545 \\
\hline 3 & 641 & 14 & 571 & 125 & 556 \\
\hline 4 & 645 & 15 & 576 & 134 & 580 \\
\hline 5 & 655 & 23 & 567 & 135 & 569 \\
\hline & & 24 & 632 & 145 & 556 \\
\hline & & 25 & 594 & 234 & 550 \\
\hline & & 34 & 578 & 235 & 548 \\
\hline & & 35 & 597 & 245 & 567 \\
\hline & & 45 & 595 & 345 & 565 \\
\hline
\end{tabular}

Note $-S P=$ serial position, $M L=$ mean latency

${ }^{*}$ Digits indicate serial positions of critical letters (serial positions numbered from left side of display to right).

$131.5, \mathrm{p}<.001)$ but show no significant variation due to redundancy in the noise letters $(\mathrm{F}=1.3, \mathrm{p}>.20)$. For negative trials, the redundancy effect is also significant $(F=10.9, p<.001)$, but there is a significant difference between the size of this effect and the size of the effect of redundant critical letters $(F=29.3, p<.001)$. As is usually found in yes-no studies of this type (Nickerson, 1972), negative responses are significantly slower than positive responses $(F=47.9, \mathrm{p}<.001)$. The error rate associated with each of the points in Fig. 1 is less than $5 \%$, so it is safe to assume that these effects are not due to variation in the proportion of guessing responses across conditions.

In order to obtain a more detailed view of the redundancy effects, it is useful to look at latencies for positive trials at each redundancy level as a function of the location of the critical letters. This information is presented in Table 1 ; positive and negative trials with redundant noise letters are not included, as no interesting trends were observed in those data. While the serial position data can be combined in a number of ways, two particular comparisons deserve special attention. Note that for the no-redundancy condition, the mean latency for the first serial position on the left is $62 \mathrm{msec}$ lower than the average latency for the other four serial positions $(t=4.46 ; p<.01)$. However, for the single-redundancy condition, the difference between latencies for displays with and without a critical letter in the first position is only $22 \mathrm{msec}$, and for the double-redundancy condition, the average latency for displays with no critical letter in the first serial position is actually about $2 \mathrm{msec}$ faster than for displays having a critical letter in that position. Thus, with no redundancy, Ss responded much faster on positive trials when the critical letter was the first letter on the left, but this effect disappears as redundancy increases.

The second observation concerning the serial position data has to do with the effect of grouping redundant 
critical letters in the display. One might expect faster latencies when redundant critical letters appear in adjacent serial positions than when they are separated by one or more noise letters; however, the data show little evidence of any strong grouping effect. Comparing adjacent to nonadjacent critical letters for the single-redundancy condition, the mean latencies differ by only $9.2 \mathrm{msec}$. For double-redundancy, the means are 569,559 , and $554 \mathrm{msec}$ for displays having no adjacent critical letters, a pair of adjacent letters, and three adjacent letters, respectively. (There are no significant differences among these means.) This observation will become more pertinent when the results of Experiment II are presented.

\section{Discussion}

It is clear from these results that even in a task with negligible error rates, response latency decreases markedly with increasing redundancy in the critical letter. This finding is inconsistent with the assumption of a simple exhaustive comparison process [such as that proposed by Atkinson et al (1969) for visual search] but can be explained in terms of primary detection responses as hypothesized by Estes (1972), since the probability of a primary detection response increases with redundancy.

Given the inadequacy of a simple exhaustive processing model, is there any way the model can be revised yet remain consistent with the data of Atkinson et al (1969)? Two of the findings in these data make it difficult to find a suitable revision. First, redundancy has a large effect only when the critical letter is redundant. This rules out any explanation in terms of decreased comparison time for redundant letters. If the redundancy effect were due solely to redundant letters being processed faster than others, then redundant noise letters should decrease mean response latency as much as redundant critical letters. One could get around this problem by assuming that critical letters are processed more rapidly than noise letters, but this brings up the second finding that gives difficulty. The mean latency for positive trials changes by $52 \pm 4.7 \mathrm{msec}$ (mean \pm standard error) when going from displays having a single critical letter to those having two. Yet, under the above assumptions, the difference between latencies in the no-redundancy condition and in the single-redundancy condition can be at most equal to the comparison time for a single noise letter. ${ }^{1}$ This latter quantity was estimated by Atkinson et al (1969) to be $25 \pm 4.3 \mathrm{msec}$. This estimate is relevant because the apparatus, exposure time, and display visual angle used in the present study were identical to those used by Atkinson et al. Clearly, the observed effect of redundant critical letters is too large to be accounted for solely by assumptions about the rate of comparison.

Another approach to finding an appropriate revision of the simple exhaustive processing model is to assume that redundancy in the critical letter has the effect of inducing some degree of self-termination. For instance, it could be assumed that Ss self-terminate with some probability upon detecting the second or third occurrence of a critical letter. The possibility of self-termination with redundant critical letters can be investigated by comparing the rate of change in mean latency for positive and negative trials as display size is varied. These rates should differ if redundancy is inducing self-termination. This comparison is carried out in Experiment II.

Yet another possibility is that Ss are able to rapidly detect the presence of redundancy per se. For example, Ss may perform a fast search for redundancy followed by classification of the redundant letter as a critical or noise letter, a positive response being made if the redundant item is a critical letter or a slower search for the critical letter being carried out if it is not. While this can be considered a type of self-terminating serach, it differs from the type of self-termination discussed above in that here two search processes are involved, one for redundancy and a second for the critical letter. The hypothesis of two searches, one fast and the other relatively slow, is similar in some ways to Estes's (1972) hypothesis of primary and secondary responses. The possibility of rapid detection of redundancy is also investigated in Experiment II.

All of the hypotheses considered above have involved revision of the comparison process in one form or another. Of course, it may be the case that some other processing stage is affected by redundancy. For instance, perhaps after completion of the comparison process the speed of response selection is affected by the number of critical letters detected in the display. This hypothesis will be further discussed after presentation of the data from Experiment II.

Up to this point, the discussion has centered on the effects of redundant critical letters. Recall that redundant noise letters also had an effect upon response latency, although the effect was relatively small and significant only for negative trials. Estes's (1972) hypotheses regarding redundancy deal only with critical letters and lead to no specific predictions concerning redundant noise letters. However, in the same paper, Estes does hypothesize that in the absence of a primary detection response, the information obtained from the items in the display is scanned for the presence of a critical letter. Considering this scan the equivalent of what has here been called the comparison process, an explanation of the complete set of redundancy effects suggests itself. If, during the scan preceding a secondary response, redundant letters (critical or noise) are processed slightly faster than others, then mean latericy on negative trials would decrease slightly with increasing redundancy. There would be less of a decrease on positive trials with noise redundant because a certain proportion of those latencies are the result of primary detection responses, and the probability of such responses should remain constant with increasing noise redundancy. Redundant critical letters would have a 
large effect on mean latency since the scanning time prior to a secondary response decreases and the probability of a primary detection response increases with increasing redundancy. Of course, the hypothesis of fast processing of redundant letters could also be combined with one of the revisions of the exhaustive search model mentioned above to account for the effect of redundant noise letters.

\section{EXPERIMENT II}

The purpose of this study is to determine, first, whether Ss can carry out a fast search for redundancy per se and, second, whether there is any evidence of self-termination when $S s$ are searching for a predesignated critical letter that is redundant in the display. The answers to these questions will provide useful information concerning the adequacy of the various proposed revisions of the simple exhaustive search model.

Cavanagh and Parkman (1972) have previously investigated response latencies in a task requiring $S s$ to search a visual display of letters for the presence of redundancy. Their data appear to indicate that a search for redundancy is slower than a search for a predesignated critical letter. However, interpretation of their data is complicated by the fact that the error rates were quite high; also, the study did not include a condition in which Ss searched for a predesignated critical letter against which to compare the search rates for redundancy. These problems are eliminated in the present study.

\section{Method}

Subjects. The Ss were 10 female Stanford University undergraduates with normal vision. Each was paid a total of $\$ 17.50$ for the 10 sessions of the experiment. In all sessions, the task was to scan a visually presented array of letters to determ ine whether or not any one of the letters was repeated in the display.

Stimuli. The display slides were prepared in the same way as in the first study. Each display contained 2, 3, 4, or 5 letters. Half the displays in each size category had 1 letter repeated, whereas the other half contained no repetitions. In addition, if a display contained a repeated letter, the 2 identical letters were located next to each other in half the displays and (except for the 2-letter slides) had 1 or more intervening letters in the other displays. In the displays in which the repeated letters were not physically grouped, all possible letter permutations were used equally often. A total of 192 different slides were made, 48 at each display size.

Procedure. The apparatus and procedure were the same as in the first study, with the exception that each session consisted of all 192 displays being presented once each in a random order. In Sessions 1-8, no letter was displayed on the Binaview unit, but a blank character served as a ready signal for $S$ to respond. In Sessions 9-10, a single letter was presented on the Binaview. This letter never appeared in the following display if no letters were repeated; however, if any letter was repeated in the display, it was the one shown previously on the Binaview. For all sessions, Ss were instructed to make a positive response if any letter was repeated in the display and a negative response otherwise. They were told that on Sessions 9-10 the critical letter presented on the Binaview would be the only one that would be repeated in the following display, if indeed there was a repetition; thus, the

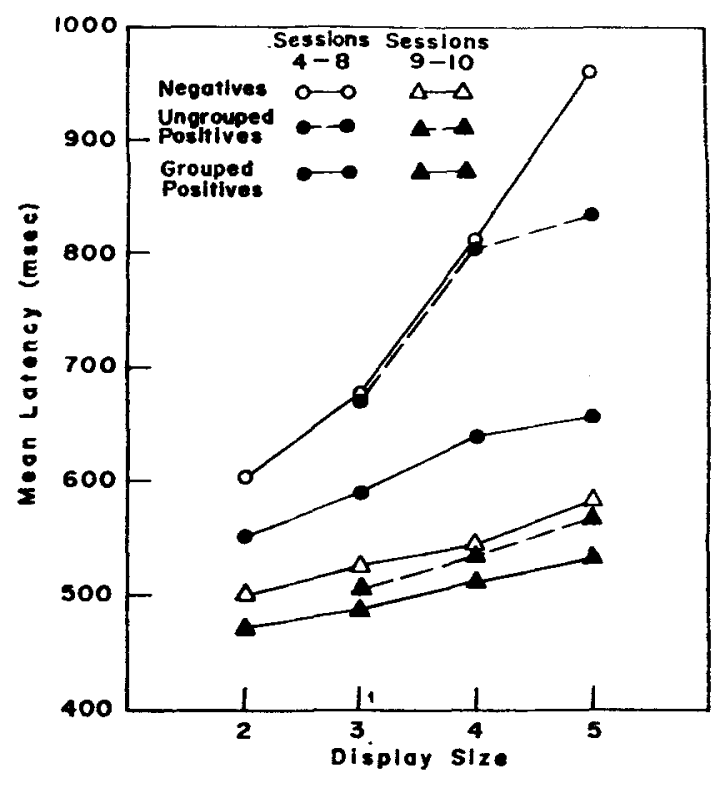

Fig. 2. Mean latency of positive (grouped and ungrouped) and negative responses for Experiment II as a function of display size (Sessions 9 and 10 shown separately).

task in Sessions 9 and 10 was identical to that of Atkinson et al (1969), except that when a critical letter appeared in the display, it was redundant.

\section{Results}

Examination of data for each session showed that mean latencies decreased over the first three sessions and were stable over the remaining sessions; therefore, the results reported here are based on Sessions 4-10. Figure 2 shows mean latencies of correct responses as a function of display size for three types of trials; these are negative, positive with adjacent redundant critical letters (grouped positives), and positive with nonadjacent redundant critical letters (ungrouped positives). Data for Sessions 9 and 10 (during which a letter was presented prior to the display on each trial) are shown separately. Error rates were under $5 \%$, with three exceptions, all in Sessions 4-8. For ungrouped positives at Display Sizes 4 and 5, the error rates were $8.1 \%$ and $13.0 \%$, respectively; for negatives at Display Size 5 , the error rate was $6.5 \%$.

Best-fitting lines were found for each of the negative and grouped positive curves in Fig. 2. The slopes and intercepts of these lines are given in Table 2. None of the curves deviate significantly from linearity. For Sessions 4-8, the negative and grouped positive slopes are significantly different $(t=7.1, p<.001)$, but for Sessions 9 and 10, these slopes do not differ significantly. Both the negative and grouped positive slopes for Sessions 4-8 differ significantly from the corresponding slopes for Sessions 9 and $10(t=7.3$, $\mathrm{p}<.001$, and $\mathrm{t}=3.3, \mathrm{p}<.05$, respectively).

Serial position data were examined, but there were no consistent trends of any interest for Sessions 4-8 (aside from the difference between grouped and ungrouped positives). The serial position data for Sessions 9 and 10 
Table 2

Slopes and Intercepts (in Milliseconds) of Lines Providing Best Fit to Latencies as a Function of Display Size

\begin{tabular}{llrr} 
& & \multicolumn{2}{c}{ Sessions } \\
\cline { 3 - 4 } & & $4-8$ & 9 and 10 \\
\hline \multirow{2}{*}{ Negative } & Slope & 120.5 & 26.0 \\
Grouped & Intercept & 338.5 & 445.5 \\
Positive & Slope & 36.1 & 21.0 \\
\hline
\end{tabular}

also showed no particular pattern of interest, although it is worth noting that for Display Size 5 the serial position data were similar to the corresponding data in Experiment $I(r=.70, p<.05)$.

\section{Discussion}

Experiment II shows quite clearly that two of the proposed revisions of the exhaustive search model are untenable. First, when searching for redundancy per se Ss were much slower than when searching for a predesignated redundant critical letter. Therefore, there is no evidence that Ss in Experiment I were searching for redundancy prior to searching for the critical letter. Second, the near-equality of positive and negative slopes for Sessions 9 and 10 goes counter to the assumption that redundancy induces self-termination.

Turning to Sessions 9 and 10 , it is interesting to note how similar the slopes found here are to the slopes obtained by Atkinson et al (1969). Their positive and negative slopes were 23.8 and $26.2 \mathrm{msec}$, respectively, compared to the slopes of 21.0 and $26.0 \mathrm{msec}$ found here. (If the grouped and ungrouped positive trials are combined, the positive slope becomes $26.2 \mathrm{msec}$.) The intercepts, however, are not that similar in the two studies (although the differences are not quite statistically significant). The Atkinson et al positive and negative intercepts were 444 and $474 \mathrm{msec}$, respectively, while those obtained here are 425 and $445 \mathrm{msec}$. (Again, if grouped and ungrouped positives are combined, the positive intercept becomes $417 \mathrm{msec}$.) The relevance of these comparisons is that in both studies (run using the same apparatus under very similar conditions), the evidence indicates exhaustive processing, with an estimated mean comparison time of about $25 \mathrm{msec}$ per item. The only apparent effect of redundancy was to lower the intercept, which in terms of the exhaustive processing model is an estimate of the duration of operations such as stimulus encoding and response selection and execution. Note, however, that both the positive and negative intercepts were lower in the present study (and by about the same amount), suggesting that redundancy may affect the time to select or execute either type of response (perhaps by making positive and negative displays more discriminable). Thus, of the various revisions of the exhaustive processing model considered in discussing Experiment $I$, the hypothesis that redundancy affects the duration of the response selection process seems best supported by the data of Experiment II. Of course, in view of the fact that the intercept differences between this study and the Atkinson et al study are not significant, these conclusions are only tentative.

What implications do the present data have for Estes' explanation of redundancy effects? With a slight elaboration of his hypotheses, it was possible to provide a good qualitative account of the results in Experiment I. It is more difficult to apply these same ideas to the data from Sessions 9 and 10 of Experiment II due to the fact that Estes makes no specific assumptions about the form of the function relating the probability of a primary detection response to display size and redundancy level. However, two of the findings in this study seem to present problems for Estes's explanation of redundancy effects. First, redundancy apparently does not affect the slope of the function relating display size to response latency; second, the intercepts for both positive and negative trials appear to be lower when all positive trials are of the single-redundancy type (as in the present study) than when they are all of the no-redundancy type (as in the Atkinson et al study). Since the effect of redundancy is assumed by Estes to be due to an increase in the number of primary detection responses and since such responses occur only on positive trials, it appears difficult to explain why redundancy does not affect the positive slope and why it does affect the negative intercept. An adequate explanation of these effects would seem to require alteration or elaboration of Estes's hypotheses regarding redundancy.

\section{REFERENCES}

Atkinson, R. C., Holmgren, J. E., \& Juola, J. F. Processing time as influenced by the number of elements in a visual display. Perception \& Psychophysics, 1969,6, 321-326.

Bjork, E. S., \& Estes, W. K. Detection and placement of redundant signal elements in tachistoscopic displays of letters. Perception \& Psychophysics, 1971, 9, 439-442.

Cavanagh, J. P., \& Parkman, J. M. Search processes for detecting repeated items in a visual display. Perception \& Psychophysics, 1972, 11, 43-45.

Estes, W. K. Interactions of signal and background variables in visual processing. Perception \& Psychophysics, 1972, 12 . 278-286.

Estes, W. K., \& Taylor, H. A. Visual detection in relation to display size and redundancy of critical elements. Perception \& Psychophysics, 1966, 1,9-16.

Nickerson, R. S. Binary-classification reaction time: A review of some studies of human information-processing capabilities. Psychonomic Monograph Supplements, 1972, 4(17, Whole No. 65), 275-318.

Wolford, G. S. Wessel, D. L., \& Estes, W. K. Further evidence concerning scanning and sampling assumptions of visual detection models. Perception \& Psychophysics, 1968, 3, 439-444.

\section{NOTE}

1. This is under the additional assumption that in the single-redundancy condition the total processing time for the pair of redundant letters is no less than the processing time for a single nonredundant letter. If this assumption is relaxed, the best estimate for these data of the time to process a pair of redundant letters is $0 \mathrm{msec}$, so the adequacy of such a model is still highly questionable.

(Received for publication October 24, 1973; revision received March 4, 1974.) 